\title{
Las microalgas: una fuente renovable para la obtención de ácidos grasos omega-3 de cadena larga para la nutrición humana y animal
}

\author{
Microalgae: A renewable source for obtaining \\ omega-3 long-chain fatty acids for \\ human and animal nutrition
}

\begin{abstract}
Omega-3 long-chain polyunsaturated fatty acids (omega-3 LCPUFA) EPA and DHA, are currently considered of nutritional importance due the numerous positive health effects observed from its administration both in human and animal nutrition. However, sources to provide these fatty acids for supplementation are not abundant, being mainly fish oil in the case of humans and fish meal and fish oil in the case of animals, the main nutritional sources of omega-3 LCPUFA. However, fish meal and fish oil are products that have begun to diminish its availability primarily due to overexploitation of the fish resource, which currently produces a high cost of these products. Some vegetable oils contain alpha-linolenic acid, which is also an omega-3 fatty acid and the precursor of omega-3 LCPUFA, but that does not replace EPA and DHA in their nutritional effects. More recently microalgae have emerged as a renewable alternative for obtaining of omega-3 LCPUFA, mainly for its composition of EPA and/or $D H A$ and good extractive yielding. These microorganisms can be artificially cultivated at industrial scale and properly processed as such (lyophilized, microencapsulated) or the oil and/or phospholipids extracted from these organisms (expended as such or microencapsulated), can be used for be added to a variety of foods for humans and animals feed (pets, animal feedlots, fish farming, among others). This paper analyzes the potential for the use of microalgae as source of omega-3 LCPUFA for human and animal nutrition.

Key words: Omega-3 LCPUFA, EPA, DHA, microalgae, human nutrition, animal nutrition.
\end{abstract}

\section{INTRODUCCIÓN}

La importancia nutricional de los ácidos grasos omega-3 está sólidamente fundamentada en la literatura científica. Se les considera nutrientes importantes en la protección de la salud cardiovascular y del sistema nervioso y visual, principalmente por sus funciones antiinflamatorias, antiarritmicas, inmunoprotectoras, citoprotectoras, neuroprotectoras y antiapoptóticas, tanto en la salud humana como animal $(1,2)$. Los ácidos grasos omega-3 más importantes son el ácido alfa linolénico (C18:3, ALN), el ácido eicosapentaenoico (C20:5, EPA) y el ácido docosahexaenoico (C22:6, DHA). A los dos últimos (EPA y DHA) se les identifica como ácidos grasos poliinsaturados de cadena larga (AGPICL) y ambos derivan del precursor ALN, el que a través de procesos de elongación y desaturación, realizado por enzimas específicas (denominadas elongasas y desaturasas), se transforma primero
Alfonso Valenzuela B. $(1,2)$

Julio Sanhueza C. (1)

Rodrigo Valenzuela B. $(1,3)$

(1) Instituto de Nutrición y Tecnología de los Alimentos Fernando Monckeberg Barros (INTA), Universidad de Chile, Santiago, Chile

(2) Facultad de Medicina, Universidad de los Andes, Santiago, Chile

(3) Departamento de Nutrición, Facultad de Medicina, Universidad de Chile, Santiago, Chile

Dirigir la correspondencia a: Profesor Alfonso Valenzuela Bonomo Instituto de Nutrición y Tecnología de los Alimentos Fernando Monckeberg Barros

(INTA), Universidad de Chile Santiago, Chile E-mail: avalenzu@inta.uchile.cl

Este trabajo fue recibido el 10 de Abril de 2015 y aceptado para ser publicado el 26 de Mayo de 2015.

en EPA y posteriormente en DHA $(1,3)$. Existe una importante diferencia en el origen del ALN y del EPA y DHA. El ALN está sólo presente en organismos vegetales de origen terrestre (semillas y frutos de oleaginosas), el EPA y el DHA solo están presentes en organismos tanto vegetales (algas y microalgas) como animales (peces, crustáceos, mariscos y algunos mamíferos) de origen marino (4). De esta forma, el beneficio derivado de los AGPICL omega-3 sólo se obtiene a partir del consumo de productos del mar (vegetales y/o animales) ya que los vegetales terrestres sólo aportan ALN (5). El efecto del aporte de ALN para conseguir el beneficio de los AGPICL omega-3 es sólo parcial porque en la mayoría de los animales la eficiencia de bioconversión del ALN en EPA y DHA es muy baja (4), por lo cual estos ácidos grasos deben ser aportados preformados, esto es a partir del consumo de organismos de origen marino (6). El desafío actual en nutrición 
tanto humana como animal es contar con una fuente adecuada, sustentable y de bajo costo de estos ácidos grasos (7). La figura 1 muestra la formación de EPA y DHA a partir del precursor ALN. Se observa la importante función de las enzimas desaturasas y elongasas en esta biotransformación.

En la nutrición animal actualmente se valoriza el aporte de AGPICL omega-3 ya que sus beneficios en la salud son similares a los observados en humanos, en los cuales se ha realizado la mayor parte de la investigación sobre estos ácidos grasos (8). De particular importancia es la alimentación a través de dietas formuladas, ya que estas fácilmente pueden ser deficitarias en uno o más nutrientes, siendo en este caso los AGPICL omega-3 los más complejos de aportar por su alta susceptibilidad al desarrollo de procesos oxidativos (rancidez) derivada de la aplicación de altas temperaturas durante el proceso de elaboración de los alimentos (9).

Este trabajo revisa en forma no exhaustiva las principales fuentes de AGPICL omega-3 disponibles para la suplementación de la alimentación tanto humana como animal, con especial énfasis en las microalgas como fuente sustentable y renovable de estos ácidos grasos, particularmente de EPA y/o DHA.

\section{Fuentes nutricionales de EPA y DHA}

Tradicionalmente, en nutrición tanto humana como animal, la fuente más común y abundante de AGPICL omega-3 para fines de suplementación han sido los aceites marinos por su alto contenido de EPA y DHA (25-30\% en promedio) (10). Se han utilizado aceites obtenidos de la pesca pelágica (sardina, anchoa, jurel, atún, entre otros) o provenientes del procesamiento de subproductos de la industria acuicultora (aceite se salmón, principalmente).

En nutrición animal, además, es utilizada la harina de pescado como fuente no sólo de proteína, también de EPA y DHA, ya que la grasa residual ( $8 \%$ ) que contiene esta harina tiene un alto contenido de AGPICL omega-3 (11).

Sin embargo, en los últimos años se ha producido una importante disminución en la captura de peces para la obtención de harina y aceite, principalmente producida por una sostenida disminución del recurso pesquero en los países con mayor actividad en este rubro (Chile y Perú en Latinoamérica), aspecto que ha producido una escasa oferta y un constante aumento del precio de estos productos (10). El uso de aceites marinos, además, trae consigo efectos nutricionales y organolépticos no deseados, como lo son la alta inestabilidad a la oxidación (por su alta poliinsaturación) y el indeseable "olor a pescado" de este producto, el que si bien puede ser desodorizado, al poco tiempo se produce la reversión del olor.

Esta situación ha motivado la búsqueda de nuevas fuentes de AGPICL omega-3 (12). Una alternativa ha sido el uso de aceites vegetales con alto contenido de ALN (canola, linaza, chía), sin embargo, como ya se comentó, la conversión del ALN en EPA y DHA es muy baja en los humanos, en los animales en general (perros, gatos, cerdos, entre otros) y también en peces de cultivo (salmón, trucha, tilapia, entre otros), con lo cual siempre existe el riesgo de deficiencia nutricional de EPA y/o DHA (10). En el caso de peces de cultivo (salmón y trucha) el remplazo parcial de la harina y el aceite de pescado, componentes alimentarios fundamentales para estos peces, por harinas y aceites de vege-

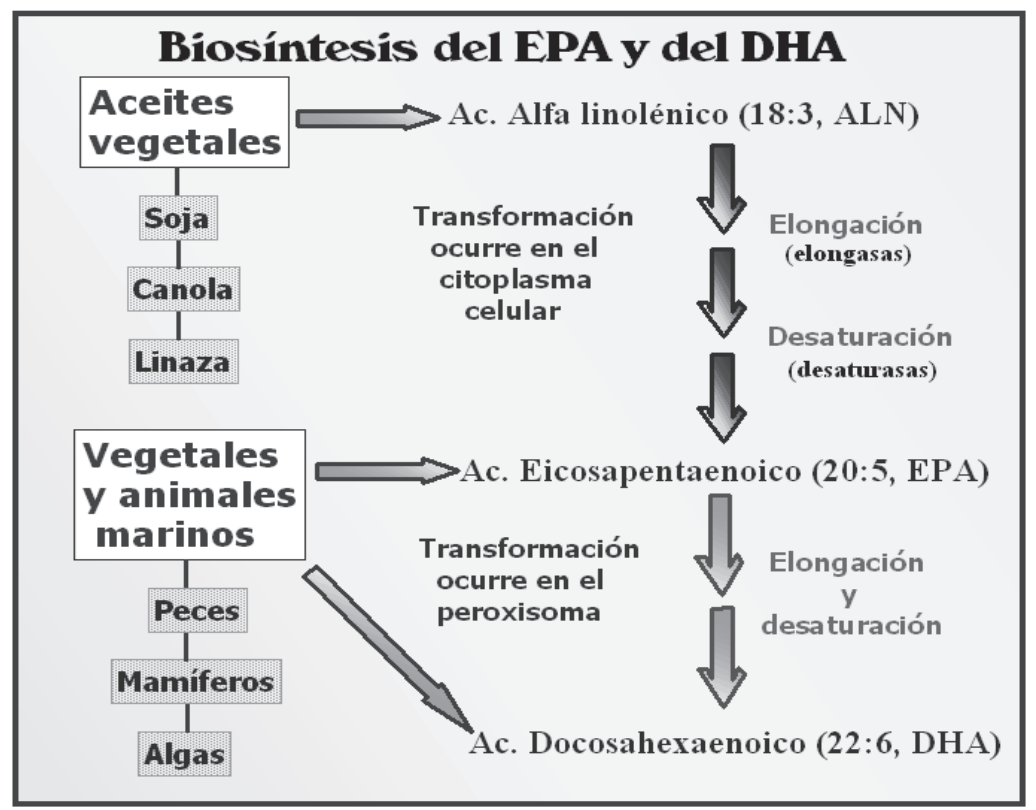

Se observa la importante función de las enzimas desaturasas y elongasas en esta biotransformación. 
tales terrestres ha tenido nefastos resultados ya que la escasa conversión del ALN en EPA y DHA en estos peces les produce desequilibrios en su respuesta inmune haciéndolos muy susceptibles a infecciones (virus ISA, por ejemplo) (12).

Estas negativas situaciones, derivadas del déficit de AGPICL omega-3, han motivado a la industria de nutrición tanto humana como animal, a realizar esfuerzos a través de investigación y desarrollo para la búsqueda de alternativas de remplazo del aceite de pescado, en el caso de los humanos y del aceite y la harina de pescado en el caso de los animales, como fuentes de AGPICL omega-3. Las microalgas pueden constituir una solución muy interesante y de gran perspectiva.

Las microalgas, una fuente renovable de AGPICL omega-3

Las microalgas, son organismos unicelulares marinos o de agua dulce microscópicos o de pequeño tamaño utilizadas desde siglos en la alimentación humana y animal. Hay antecedentes que la spirulina, una microalga, era consumida por los aztecas en México hace más de 500 años, aspecto que fue descrito por los cronista españoles. Sin embargo, el uso de otras fuentes alimentarias, principalmente proveedoras de proteínas, redujo notablemente el consumo y el interés por estos microorganismos (13).

Recientemente, el mejor conocimiento de la composición química y del valor nutricional de varias especies de microalgas y la posibilidad de realizar su cultivo en forma artificial, ha despertado un creciente interés por estos microorganismos como una importante fuente de nutrientes, particularmente de AGPICL omega-3. La microalgas pueden ser fotoautótrofas (fotosintéticas) para lo cual solo requieren de la presencia de luz y CO2 para multiplicarse, o en el otro extremo, pueden ser heterótrofas, para lo cual requieren de compuestos orgánicos más complejos para su nutrición. Sin embargo, se ha identificado una gama amplia de combinaciones de estas modalidades de supervivencia, ya que existen microalgas fotoheterótrofas (requieren de luz y de la presencia de compuestos orgánicos) y mixótrofas (pueden crecer indistintamente en forma autótrofa o heterótrofa, dependiendo de las condiciones del medio ambiente). La figura 2 muestra las diferentes modalidades de crecimiento de las microalgas.

Cualquiera que sea la condición de requerimientos de las microalgas, estas se reproducen con gran rapidez (una generación cada 20 o 30 minutos) con lo cual cuando son cultivadas en un ambiente adecuado pueden generar una gran cantidad de biomasa en pocas horas. Actualmente se multiplican en foto o bioreactores de alta eficiencia y en condiciones controladas (temperatura, $\mathrm{pH}$, nutrientes, entre otros) que aseguran la pureza del producto (ausencia de contaminación) y la eficiencia productiva (14). El análisis de su composición química indica un importante aporte de proteínas, polisacáridos, micro y macrominerales, vitaminas, una variedad de productos bioactivos (carotenoides, fitoesteroles, polifenoles, entre otros) y en algunas de ellas, de AGPICL omega-3 EPA y/o DHA.

Microalgas, como Schizochytrium SP, Phaeodactylum tricornutum o Isochrysis galbana, acumulan EPA y DHA en la forma de micro gotas citoplamáticas de triglicéridos (aceite) y también en los fosfolípidos de sus membranas (plasmática y retículos) $(15,16)$. Este aspecto es tremendamente relevante ya que actualmente se valoriza más el aporte de AGPICL omega-3 en la forma de fosfolípidos que de triglicéridos por dos razones importantes: la biodisponibilidad de los AGPICL omega-3 en el sistema digestivo es mucho mayor cuando se aportan como fosfolípidos que cuando se aportan como triglicéridos (aceites) (17); los fosfolípidos, además de aportar ácidos grasos omega-3, también aportan otros nutrientes importantes, como colina (a partir de fosfatidilcolina), inosiltol (fosfatidilinositol), serina

\section{Tipos de microalgas}

Fotoautótrofas: Obtienen energía de la luz solar, son fotosintéticas

Fotoheterótrofas: Obtienen energía de la luz solar y también de compuestos orgánicos

Mixótrofas: Pueden crecer en forma autótrofa $o$ en forma heterótrofa

Heterótrofas: Requieren compuestos orgánicos para su nutrición, crecen en ausencia de luz 
(fosfatidilserina), entre otros $(12,18)$. De esta forma, el aporte de AGPICL omega-3 en la forma de fosfolípidos entregaría un doble beneficio nutricional.

En base a lo expuesto, se concluye que las microalgas pueden ser una muy interesante alternativa ya que además de aportar macro y micronutrientes, algunas de ellas serían una fuente muy adecuada de AGPICL omega-3, principalmente de DHA, en la forma de triglicéridos y de fosfolípidos de alta biodisponibilidad, en forma limpia, eficiente y renovable. Estas características transforman a las microalgas en una modalidad de suplementación nutricional para la alimentación tanto humana como animal altamente costo/eficiente (13).

Las microalgas se pueden utilizar como tal liofilizadas (19), microencapsuladas (20), se les puede extraer el aceite y fosfolípidos (21) y tanto el aceite como los fosfolípidos pueden ser separados y microencapsulados (22). La figura 3 muestra una comparación en el aporte de AGPICL omega-3 a partir de microalgas, del pescado (harina y aceite) y del krill, un pequeño crustáceo que se captura en los mares antárticos y que también constituye una muy buen fuente de AGPICL omega-3 de alto costo, principalmente en la forma de fosfolípidos (23), pero su captura está sujeta a regulación internacional porque es el principal alimento del ecosistema antártico, con lo cual no es una alternativa económicamente viable actualmente y en el futuro.

\section{RESUMEN}

Los ácidos grasos polinsaturados omega-3 de cadena larga (AGPICL omega-3) EPA y DHA, son considerados actualmente de gran importancia nutricional debido a los numerosos efectos positivos en la salud observados a partir de su administración tanto en la nutrición humana como animal, por lo cual se sugiere su suplementación. Sin embargo, las fuentes para aportarlos como suplemento no son muchas, siendo principalmente el aceite de pescado, en el caso de los humanos, y la harina y el aceite de pescado en el caso de los animales, la principal fuente de suplementación de AGPICL omega-3. Tanto el aceite de pescado como la harina de pescado son productos que han comenzado han disminuir su disponibilidad debido, principalmente, a la sobreexplotación del recurso pesquero, con lo cual ambos productos tienen actualmente alto valor. Algunos aceites de origen vegetal contienen ácido alfa linolénico, el cual es un ácido graso omega-3 y el precursor de los AGPICL omega-3, pero que no remplaza en sus efectos nutricionales al EPA y DHA. Más recientemente, las microalgas han surgido como una alternativa renovable para la obtención de AGPICL omega-3, principalmente por su aporte de EPA y/o DHA y buen rendimiento de extracción. Estos microorganismos pueden ser cultivados artificialmente a escala industrial y debidamente procesados (liofilizados, microencapsulados, etc.) o el aceite y/o los fosfolípidos extraídos de ellos (consumidos como tal o microencapsulados), puede ser utilizados para adicionarse a una variedad de alimentos tanto para humanos como para animales (mascotas, animales de engorda, peces en cultivo, entre otros). Este trabajo analiza la potencialidad del uso de las microalgas en la nutrición humana y animal como fuentes de AGPICL omega-3.

Palabras clave: AGPICL omega-3, EPA, DHA, microalgas, nutrición humana, nutrición animal.

\section{BIBLIOGRAFIA}

1. Brenna J, Salem N, Sinclair A, Cunnane S. -Linolenic acid supplementation and conversion to n-3 long-chain polyunsaturated fatty acids in humans. Prostaglandins, Leukot Essent Fatty Acids, 2009; 80: 85-91.

2. Brenna J, Carlson S. Docosahexaenoic acid and human brain development: Evidence that dietary supply is

\section{Contenido de AGPICL omega -3 EPA y DHA proveniente de difierentes fuentes}
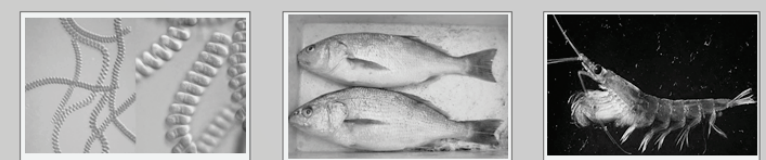

\begin{tabular}{|c|c|c|}
\hline Microalgas & Pescado & Krill \\
\hline $3 \%$ EPA & $14 \%$ EPA & $30 \%$ EPA \\
\hline $35 \%$ DHA & $12 \%$ DHA & $9 \%$ DHA \\
\hline
\end{tabular}

Modificado de Ref. (15) 
needed for optimal development. J Hum Evol. 2014; 77: 99-106.

3. Calder P. Omega-3 polyunsaturated fatty acids and inflammatory processes: nutrition or pharmacology? Brit J Clin Pharmacol. 2012; 75: 645-62.

4. Swanson D, Block R, Mousa S. Omega-3 fatty acids EPA and DHA: Health benefits throughout life. Adv Nutr. 2012; 3: 1-7.

5. Larsen R, Eilertsen $K$, Elvevoll E. Health benefits of marine foods and ingredients. Biotechnol Adv. 2011; 29: 508-18.

6. Valenzuela A, Sanhueza J, Nieto S. Docosahexaenoic acid (DHA), essentiality and requirements: Why and how to provide supplementation. Grasas Aceites. 2006; 57: 229-37.

7. Taneja A, Singh H. Challenges for the delivery of long-chain fatty acids in functional foods. Ann Rev Food Sci Technol. 2012; 3: 105-23.

8. Mozaffarian D, Wu J. (n-3) Fatty acids and cardiovascular health: Are effects of EPA and DHA shared or complementary? J Nutr. 2012; 142: 614S-25S.

9. Valenzuela A. Aceites de origen marino y su importancia en la salud y nutrición humana y animal. Aceites Grasas. 2009; XIX: 154-61.

10. Valenzuela A, Sanhueza J, De la Barra F. El aceite de pescado: ayer un desecho industrial, hoy un producto de alto valor nutricional. Rev Chil Nutr. 2012; 39: 201-9.

11. Valenzuela $A$, Valenzuela $R$, Sanhueza J, De la Barra $F$, Morales G. Fosfolípidos de origen marino: una nueva alternativa para la suplementación con ácidos grasos omega-3. Rev Chil Nutr. 2014; 41: 433-8.

12. Valenzuela A, Valenzuela $R$. Ácidos grasos omega-3 en la nutrición ¿Cómo aportarlos? Rev Chil Nutr. 2014; 41: 205-11.

13. Adarme-Vega C, Lim D, Timmins $M$, et al. Microalgal biofactories: a promising approach towards sustainable omega-3 fatty acid production. Microbial Cell Fact. 2012; 11: 96-105.

14. Bahadar A, BilalKhan M. Progress in energy from microalgae: A review. Renewable and Sustainable Energy Rev 2013; 27: 128-48.

15. Burri L, Hoem N, Banni S, et al. Marine omega-3 phospholipids: metabolism and biological activities. Int J Mol Sci. 2012; 13: 15401-19.

16. Fradique $M$, Batisra A, Nunes $M$, Gouveia $L$, Bandarra $N$, Raymundo $A$. Isochrysis galbana and Diacronema vilkaianun biomass in pasta products as PUFA's source. Food Sci Nutr Technol. 2013; 50: 312-9.

17. Schuchardt C, Hahn A. Bioavailability of long-chain fatty acids. Prostaglandins Leukot Essent Fatty Acids. 2013; 89: 1-8.

18. Parmentier M, Al Sayed M, Linder M, Fanni J. Polar lipids: n-3 PUFA carriers for membranes and brain: nutritional interest and emerging process. OCL 2007; 14: 224-9.

19. Draaisma $R$, Wijffels $R$, Slegers P, Brenter $L$, Roy A, Barbosa M. Food commodities from microalgae. Curr Opin Biotechnol. 2013; 24: 169-77.

20. Desai H, Parks H. Recent developments in microencapsulation of food ingredients. Drying Technol. 2005; 23: 1361-94.

21. Dejoye C, Abert M, Chamet $F$. New procedure for extraction of algal lipids from wet biomass: A green clean scalable process. Biores Technol. 2013; 134: 271-5.

22. Drush S, Benedetti S, Scampicchio M, Mannino S. Stabilisation of omega-3 fatty acids by microencapsulation. Agro Food Industry Hi-Tech. 2008; 19: 31-2.

23. Tou J, Jaczynsky J, Chen Y. Krill for human consumption: nutritional value and potential health benefits. Nutr Rev. 2007; 65: 63-77. 